Voix et Images

volxetimages

\title{
Le Téléviseur vide ou comment lire l'Antiphonaire
}

\section{Robert Mélançon}

Volume 3, numéro 2, décembre 1977

Victor-Lévy Beaulieu

URI : https://id.erudit.org/iderudit/200105ar

DOI : https://doi.org/10.7202/200105ar

Aller au sommaire du numéro

Éditeur(s)

Les Presses de l'Université du Québec

ISSN

0318-9201 (imprimé)

1705-933X (numérique)

Découvrir la revue

Citer cet article

Mélançon, R. (1977). Le Téléviseur vide ou comment lire l'Antiphonaire. Voix et Images, 3(2), 244-265. https://doi.org/10.7202/200105ar d'utilisation que vous pouvez consulter en ligne.

https://apropos.erudit.org/fr/usagers/politique-dutilisation/ 


\section{Le Téléviseur vide ou comment lire l'Antiphonaire}

\section{L'écrivain et son double}

Peu d'écrivains accordent autant d'importance à leur lecteur qu'Hubert Aquin, au point d'en ressentir comme une obsession la présence quasi fantômatique:

Jusqu'à présent, j'ai toujours imaginé le lecteur au-dessus de mon épaule, en train de me dechiffrer pendant que j'écris'.

Telle déclaration péremptoire pourtant (“je n'ai jamais pensé que j'écrivais pour moi, j'écris pour le lecteur ${ }^{2}$ ) n'est pas aussi transparente qu'il y paraît. Car si Aquin oriente complètement son travail en fonction de celui qui le lira, c'est pour lui rendre "la lecture quasiment impossible ou à tout le moins difficile", instaurant ainsi une relation ambiguë qu'il ne craint pas de pousser à ses ultimes conséquences, jusqu'à se réjouir de perdre des lecteurs découragés par la complexité du texte qui leur est proposés. II serait superficiel toutefois d'en conclure qu'Aquin ne songe à son public que pour l'agresser ou s'en débarrasser et que la difficulté de ses romans ne vise qu'à le décourager: l'exclusion de certains lecteurs n'est qu'un moment de la recherche passionnée d'un lecteur avec lequel, enfin, pourrait s'établir un rapport authentique. La difficulté prend valeur d'épreuve initiatique.

Ce semblable, ce frère, Aquin l'a maintes fois évoqué. II l'a imaginé "portant un masque dont il pourrait même se servir comme signet ${ }^{4}$, ébauche d'un rite susceptible de manifester de façon tangible une complicité fervente. II l'a invité à considérer la lecture comme "une expérience de jouissance" sans laquelle "la littérature n'a pour ainsi dire aucun intérêt ${ }^{5}$. II l'a défini comme son collaborateur nécessaire:

Dans et par la lecture, l'écrivain et le lecteur se trouvent unis l'un à l'autre et ils participent, dans un synchronisme extra-temporel, à une célébration muette. Ainsi, la littérature existe pleinement non pas quand l'cuvre est écrite, mais quand un lecteur remonte le cours des phrases et des mots pour devenir, par ce moyen, co-créateur de l'œuvre6.

C'est que l'écrivain seul s'avère impuissant à compléter le texte, qu'il ne peut vraiment devenir écrivain sans entrer en relation avec son double, sans 
l'intervention de ce lecteur «capable [...] de jouer le jeu ${ }^{7}$. Celui qui se plonge dans un roman d'Aquin ne se trouve pas convié à revivre les aventures inoffensives de personnages auxquels il s'identifierait momentanément, ni à réitérer les péripéties d'un récit achevé, clos. II n'a pas à découvrir une signification formée à l'avance, sortie toute armée de la tête de son auteur comme Minerve de celle de Jupiter, et déposée au creux du texte comme un trésor enfoui dans le sable d'une plage ${ }^{8}$. II est mis au défi de faire fonctionner une machine à fabriquer du sens. Loin donc de se définir comme réceptivité plus ou moins passive, la lecture se propose comme «écriture inversée ${ }^{9}$ " dans laquelle la fidélité au texte ne se marque pas tant par l'élucidation d'un sens déjà donné que par la description et la mise en ceuvre des règles du jeu dont l'écrivain convient avec son partenaire lecteur.

\section{Chant alternatif}

S'absorber de cette façon dans l'Antiphonaire, c'est se trouver confronté à un texte très touffu, labyrinthique, piégé à chaque page. Les rebondissements incessants de l'intrigue et les correspondances très appuyées qui s'établissent entre les événements et les personnages définissent un univers d'une complexité extrême. Deux histoires différentes par les lieux et par les époques sont racontées parallèlement, entrecroisées, contrariées l'une par l'autre ${ }^{10}$. Très sommairement, on peut résumer leurs arguments de la façon suivante, en les distinguant temporairement pour simplifier.

(A) Christine Forestier, après avoir abandonné la pratique de la médecine depuis une dizaine d'années, a entrepris une thèse de doctorat en philosophie des sciences sur l'ceuvre d'un médecin du XVIe siècle, Jules-César Beausang. En mars 1969, au cours de vacances en Californie, son mari Jean-William est victime d'une crise d'épilepsie marquée d'un paroxysme de violence: il l'attaque avec fureur, au point qu'elle doit s'enfuir de leur motel et qu'elle va chercher refuge dans une pharmacie. Le pharmacien profite de son trouble pour la droguer, la violer et la séquestrer durant une nuit. Ellè réussit à s'enfuir le lendemain matin et elle assiste de loin, sans être vue, à l'assassinat du pharmacien par Jean-William. Terrorisée, craignant qu'un pareil sort ne devienne bientôt le sien, elle retourne en hâte au motel pour récupérer ses effets personnels et elle s'enfuit, se cachant quelques jours au Holiday Inn de San Modesto pour se refaire un visage décent avant de rentrer seule à Montréal en avion, via Chicago d'où elle tentera en vain de téléphoner à Robert Bernatchez avec lequel elle a déjà entretenu une liaison épisodique et orageuse qu'elle espère ranimer. Entretemps Jean-William rentre également à Montréal, mais en voiture, et il téléphone à chaque étape à Suzanne Bernatchez à laquelle il raconte de façon délirante la liaison de Robert et de Christine. Celleci arrive à Montréal un jour avant lui et renoue avec Robert Bernatchez qui annonce aussitôt à Suzanne son intention de divorcer; elle passe au domicile conjugal pour reprendre ses effets personnels quelques heures 
à peine avant que Jean-William n'y arrive et elle s'installe chez Robert quelques jours plus tard, après que Suzanne eût quitté les lieux. Cette dernière, officiellement réfugiée chez sa sœur à Granby, s'est en fait établie chez Albert Franconi avec lequel elle avait ébauché une liaison deux ans plus tôt et qui, lui-même divorcé depuis quatre ans, vient de réussir à retracer en Californie son ancienne femme qui s'était enfuie avec leurs deux enfants; en compagnie de Suzanne, il fera un vain voyage dans la banlieue de San Francisco pour tenter de les retrouver et de les ramener à Montréal. Christine (qui a découvert qu'elle était enceinte du pharmacien qui l'avait violée à San Diego, ce qu'elle n'a pas osé avouer, mettant cet enfant sur le compte de ses relations conjugales avec Jean-William) et Robert vivent des semaines ternes, coupées de brefs moments d'exaltation, de querelles, de crises. Un soir de juin, Jean-William tente, devant Christine, d'assassiner Robert au moment où, rentrant de son travail, ce dernier arrive à son appartement. Très grièvement blessé de deux balles à la tête, il est conduit par l'ambulance de la police de Montréal (à laquelle Christine, sans pouvoir s'expliquer son attitude, omet de dénoncer Jean-William) à l'hôpital de Cartierville où il demeure inconscient, entre la vie et la mort, durant quatre jours. II est soigné par le docteur Albert Franconi, qui offre son bureau à Christine pour qu'elle puisse rester à l'hôpital en attendant d'être fixée sur le sort de Robert. Un matin, elle provoque le docteur Franconi qui l'a involontairement surprise à son réveil et elle se laisse violer en protestant faiblement, de façon très ambiguë. Jean-William, toujours en liberté, tente à nouveau d'assassiner Robert dans sa chambre d'hôpital; il échoue grâce à l'intervention d'une infirmière, réussit à s'enfuir et, cette fois, Christine ne peut pas éviter de le dénoncer au policier qui menace de l'accuser de complicité. Elle s'abandonne à une tristesse sans fond et perd peu à peu contact avec la réalité. Le docteur Franconi lui apprend, au moment où ils font de nouveau l'amour dans son bureau, que Robert, s'il guérit, sera physiquement diminué, paralysé, impuissant. Désespérée, elle se suicide la nuit suivante. Albert Franconi apportera chez lui son manuscrit, que Suzanne lira et auquel elle ajoutera une postface où l'on apprendra que Jean-William s'est suicidé lui aussi et que Robert survit, atrocement diminué. Enfin, Albert Franconi, découvrant par hasard que Suzanne a lu le journal de Christine et qu'elle sait par conséquent qu'il l'a trompée, ne peut supporter sa honte et se suicide à son tour après avoir écrit à Suzanne une lettre d'adieu, en date du 19 août 1969, qui clôt le roman.

(B) Au printemps 1536, Jules-César Beausang, médecin à Gand, disciple de Paracelse et converti à la Réforme, quitte sa femme qui le trompe et se met en route pour l'Italie où il vient d'expédier le manuscrit d'un audacieux traité de médecine à un imprimeur de Chivasso, près de Turin. Ce manuscrit est introduit clandestinement en Italie par Renata Belmissieri, une jeune épileptique qui a fui sa famille parce que ses parents la croyaient possédée du démon et qui, après avoir vécu d'expédients, a été initiée à la contrebande de textes suspects par l'abbé Leonico Chigi de Turin. J.-C. Beausang, en route pour Chivasso, fait un voyage mouve- 
menté: dévalisé par des voleurs qui ne lui laissent que le papier sur lequel il tient son journal, il pratique son art quelque temps chez un médecin de Bienne pour se renflouer financièrement puis il dévalise ce collègue pour reprendre plus vite, déguiisé en ambassadeur vénitien, son itinéraire vers l'Italie à travers les cantons suisses dont les paysages l'émerveillent. II arrive à Chivasso quelques heures avant Renata Belmissieri qui a été victime d'une crise d'épilepsie en transportant le manuscrit; il s'installe dans une auberge où il meurt de la peste après avoir fait le bilan ultime de sa vie dans son journal. Pendant ce temps, Renata qui arrive en pleine nuit à l'imprimerie où elle doit livrer le manuscrit se fait violer par l'imprimeur Carlo Zimara alors qu'elle est la proie d'une autre crise d'épilepsie; Antonella Zimara, sa femme, surprend la scène, tue son mari et s'enfuit avec Renata en emportant le manuscrit de J.-C. Beausang. Les deux femmes se réfugient auprès de l'abbé Leonico Chigi qui, après avoir entendu la confession d'Antonella, viole lui aussi Renata en proie à une troisième crise d'épilepsie dans la sacristie. Antonella, mue par la jalousie, va dénoncer Renata à la police turinoise comme l'auteur du meurtre de son mari; l'abbé Chigi, à l'endroit duquel elle exerce un certain chantage, corrobore cette accusation. Après l'arrestation de Renata, Antonella et l'abbé Chigi qu'elle a séduit et qui emprunte l'identité de Jules-César Beausang dont il emporte le manuscrit qu'il compte monnayer; s'enfuient ensemble à Genève où ils espèrent refaire leur vie. Dans cette ville, Chigi-Beausang travaille dans une imprimerie, trompé par Antonella qu'il ne peut satisfaire et qui, sous prétexte d'aider les pauvres de la ville pour justifier ses fréquentes absences, se livre sans doute à la prostitution. Après les débuts exaltés de leur vie commune, ils mènent des existences ternes, de plus en plus séparées, finissant presque par ne plus se voir, au grand désespoir de Chigi-Beausang qui devient la proie d'un irrépressible taedium vitae. En rentrant chez lui à l'improviste un après-midi, il surprend Antonella au lit avec un inconnu; il la tue, blesse l'inconnu, et s'enfuit en France sous le nom de Leonico Zimara. II aboutit à Lyon où il travaille encore dans un atelier d'imprimerie et où il publie le manuscrit de Jules-César Beausang auquel il a intégré son propre journal. II y meurt en 1537, victime d'une syphilis contractée à la fréquentation de prostituées bohémiennes.

Dans l'Antiphonaire, ces deux récits ne sont pas simplement juxtaposés l'un à l'autre comme un résumé pourrait en donner l'illusion. Ils s'interpénètrent et fusionnent en une seule fiction décentrée grâce au point de vue de narration adopté dès le deuxième chapitre (p. 17 - les chapitres ne sont pas numérotés) et maintenu de façon plus ou moins vraisemblable tout au long du roman jusqu'à la postface (p. 243). Entre l'ouverture que constitue le premier chapitre et cette conclusion, le roman se présente comme le dossier où sont rassemblés les différents textes écrits par Christine Forestier à partir du neuvième jour de ses vacances en Californie: mémoires, journal intime, fragments de sa thèse de doctorat, citations de l'ouvrage de Jules-César Beausang et des passages interpolés par Leonico Chigi, reconstitutions des événements qui se sont passés en 1536, affabulations diverses. La distinction des deux récits perd quelque peu de sa 
netteté dans cette fragmentation extrême qui n'est pas gratuite dans la mesure où chaque récit, considéré isolément, ne peut être dit complet que de façon très superficielle, au seul plan de l'intrigue dessinée à grands traits, telle qu'elle apparaît dans un résumé. Ils restent en profondeur très lacunaires. Le récit $B$, entaché d'innombrables invraisemblances de détail, par exemple d'anachronismes, serait un très médiocre roman historique s'il prétendait à la reconstitution du passé; et le récit $A$, non moins invraisemblable sous certains rapports, par exemple la caractérisation des personnages ou l'enchaînement des événements, ne peut être considéré comme le déploiement d'un univers romanesque consistant. lls forment par contre ensemble, par leur éclairage réciproque, un authentique roman dans la mesure où les lacunes de l'un trouvent leur réponse dans l'autre. On peut toutefois se méprendre sur la nature et les modalités des rapports qui les lient. Leur entrecroisement systématique, les passages incessants de l'un à l'autre et de troublants recoupements entre les événements de 1536 et ceux de 1969 créent un étourdissant jeu de miroirs qui incite le lecteur, dans un premier temps, à chercher des correspondances précises d'événement à événement, de personnage à personnage, dans l'espoir de réduire la complexité de cette double histoire. Cette recherche se trouve cependant bientôt déçue dans la mesure où la prolifération indéfinie de ces rapports binaires leur retire toute portée pratique. Jean-William Forestier, frappé d'épilepsie, répond bien à Renata Belmissieri, elle aussi épileptique; mais celle-ci, lorsqu'elle se fait violer par l'imprimeur Carlo Zimara dans l'atelier de son imprimerie puis par l'abbé Leonico Chigi dans la sacristie de son église, s'identifie à Christine Forestier, violée, elle aussi, par le pharmacien J.-L. Gordon dans le San Diego Drugstore puis par le docteur Franconi dans son bureau à l'hôpital de Cartierville; mais Christine, écrivain et médecin, reprend aussi le personnage d'un autre médecin écrivain, J.-C. Beausang, tout en s'identifiant à Leonico Chigi, lecteur, éditeur et commentateur de ce dernier dont elle est, elle aussi, lecteur et commentateur puisqu'elle prépare une thèse de doctorat sur son cuvre; mais le nombre de ses partenaires vénériens la rapproche sans conteste de l'équivoque Antonella Zímara qui se livre à la prostitution à Genève, rapprochement peu flatteur que son amant, Robert Bernatchez, ne manquera pas de souligner au cours d'une querelle; mais celui-ci, victime de deux tentatives de meurtre, réflète tout autant Carlo Zimara qui meurt assassiné par sa femme Antonella, que cette dernière, assassinée par Leonico Chigi en proie comme Jean-William à une jalousie irrépressiblement vengeresse. En poursuivant avec quelque persévérance la recherche de tels rapports, on est conduit à des conclusions apparemment insoutenables, à l'hypothèse au premier abord absurde que Jean-William, Christine, Robert, Renata, Chigi, Antonella et les autres ne sont que les avatars d'un seul et unique personnage. C'est pourtant à partir de tels recoupements qu'on a pu proposer récemment une lecture fantastique, d'inspiration borgésienne ${ }^{11}$, du roman d'Aquin. II n'y aurait qu'une seule série d'événements dans l'Antiphonaire et, en la rétablissant “dans son déroulement chronologique», on verrait que «tout découle du premier viol commis par Carlo Zimara le 9 août 
1536 ” et que "ce premier forfait entraîne tous les autres "; l'Antiphonaire développerait le “thème de la réincarnation expiatoire» et les événements de 1969 auraient pour fonction de compenser en les réitérant ceux de $1536^{12}$. Quoique cette interprétation soit extrêmement séduisante et qu'elle suggère une relecture passionnante du roman, on ne peut la considérer comme vraiment satisfaisante; elle implique une redistribution contestable des événements (l'ordre chronologique serait «abusivement perturbé par Christine ${ }^{13}$ ") et elle conduit à négliger certains aspects essentiels du texte: la variation systématique des registres d'écriture, les outrances volontaires dans l'enchaînement des actions aussi bien que dans l'expression et la constante mise en relief des artifices de la fiction. Les rapports semblent donc devoir être cherchés plutôt entre les deux récits pris en bloc. Les événements du premier ne reproduisent pas dans le détail ceux du second et la relation s'effectue, sur le mode d'une distance ironique, d'un récit à l'autre plutôt qu'entre tel ou tel de leurs aspects isolés. Juxtaposés l'un à l'autre, ils se trouvent croisés systématiquement de façon à ce que le lecteur passe sans cesse de l'un à l'autre, les lise en alternance ou, mieux, parallèlement, comme un ensemble à la fois un et double dont les perspectives ne cessent de se déplacer.

Les deux récits projettent l'un sur l'autre un éclairage constant et si, sous certains rapports, ils semblent le redoublement à deux époques et dans deux milieux différents d'une seule et unique histoire archétypale, ils n'en restent pas moins distincts. Le titre (qu'un commentateur pressé a déclaré sans signification et de pure convention ${ }^{14}$ ) désigne le mode de composition qui permet cette ambiguïté: “antiphonaire ", c'est-à-dire chant alternatif, roman à deux voix ${ }^{15}$. Cette alternance des deux histoires et la multiplication des symétries peut faire penser qu'Aquin a tenté d'exprimer une conception cyclique de l'histoire ${ }^{16}$, mythe platonicien de la grande année ou vision nietzschéenne de l'éternel retour. Dans cette hypothèse, ce serait la forme même du roman, et elle seule, qui manifesterait un sens qu'Aquin ne désigne pas autrement, que son texte ne dit pas expressément. L'interprétation séduit en ce qu'elle confère à la structure du roman une nécessité absolue, la composition alternée devenant le chiffre d'une allégorie. Elle ne la justifie toutefois que dans ses grandes masses et la complexité du texte, qui se révèle structuré dans ses moindres détails, appelle une lecture plus élaborée. Les deux voix du roman font plus que se répondre en alternant, elles s'interpénètrent pour engendrer une troisième voix, un super-récit qui assume tous leurs aspects, même les plus contradictoires, et qui les réoriente.

\section{Prosodie}

Une étude du “Plan partiel de l'Antiphonaire" qu'a publié Aquin ${ }^{17}$ montre en effet que la seule idée de récits alternés ne donne qu'une vision grossièrement schématique de la composition du roman. Au départ, ni les noms, ni les caractères des personnages, ni les événements qui leur permettront de prendre corps ne sont fixés. Ceux qui deviendront Christi- 
ne et Jean-William Forestier se réduisent à quelques indications très sommaires:

I - Introduction de l'auteur

(théorie du roman de H.A.)

(placer à la fin)?

II - Plan de l'ouvrage (schéma fait par l'Auteur).

III - La femme (Jeanne): considérations sur le XVI ${ }^{\ominus}$ siècle.

IV - Suite: début du roman impliquant le personnage masculin (W. Herbert): début brusque.

$\mathrm{V}$ - Jeanne reprend le thème et varie, changeant de gamme, de tonalité, de registre, d'instrument, de points de référence... (...)

$\mathrm{VI}$ - FB - Jeanne et $\mathrm{W}$. Herbert Jutland se prélassent quelque part en Californie (...) ${ }^{18}$

De même Jules-César Beausang n'émerge que très lentement d'une sorte de chaos où diverses possibilités sont successivement mises à l'essai:

... Un célèbre docteur dont les ouvrages ont été interdits en France... ou alors: ce serait un penseur comme Calvin...

... L'auteur (une sorte d'Érasme - exilé)...

... L'auteur est un Français de la région de Lille (Calvin)... forcé d'émigrer par des pressions cléricales... Ėtre austère et morose, il se désole sur les quais du Rhin à Bâle, errant tout au long des journées... ne sachant plus que penser...

XXII - Jules-César Beausang a repris la route: il arrive maintenant par les sentiers à Bienne, sur le lac de Bienne. II contourne ce lac, pâmé devant sa splendeur... gavé de tant de beautés naturelles... ${ }^{19}$.

Une étude qui suivrait toutes les métamorphoses de ces personnages, depuis la rêverie qui suscite leur première émergence confuse jusqu'au texte du roman où ils prennent leur forme ultime, pourrait faire considérablement progresser notre connaissance de l'imaginaire aquinien en montrant sa progression vers des formes jugées de plus en plus adéquates. II n'est pas indifférent, par exemple, que celui qui était au départ un médecin proscrit, après avoir été un moment un théologien réformateur ( un penseur comme Calvin»), puis un écrivain humaniste ( $u$ une sorte d'Érasme»), redevienne à la fin, invinciblement, médecin proscrit tout en assimilant les principaux traits de ses formes intermédiaires: Jules-César Beausang (quel nom pour un médecin et quel prénom pour un humaniste!) adhère à la Réforme et manifeste dans ses ouvrages des préoccupations encyclopédiques typiquement humanistes. II semble qu'Aquin procède par additions, qu'il cherche à intégrer dans une forme englobante des conceptions distinctes à l'origine. Et sans doute faudrait-il aussi s'interroger, à un autre niveau, sur la fascination que paraissent exercer sur lui la médecine et la pharmacie présentes dans presque tous ses textes. Mais le «Plan partiel... » présente avant tout l'intérêt d'une "sorte de combinatoire qui agence tout l'ensemble ${ }^{20}$ " et qui signale les grandes lignes de force du roman a travers leur genèse laborieuse. Au départ, la structure générale ne semble guère plus claire que la figure des divers personnages, et les deux voix de ce chant alternatif ne se fixent qu'après de longues hésitations. Le plan qui a été publié ne garde trace que de deux hypothèses, mais on a toutes raisons de penser qu'il y en eût d'autres, à peine formulées peut-être: 
1) William - Jeanne - Robert - Louise

San Diego - Montréal

(1970)

2) La jeune fille - l'imprimeur - l'épouse

Turin - Pontassieve

(16ème s.)

3) L'auteur du manuscrit

Bâle - Zurich - Neuchâtel - Lille

(16ème s. ${ }^{21)}$

AXES TEMPORELS:

1) Christine - William

Montréal 1967 et 1970

San Diego 1969

2) Renata Belmissieri

Frontière Suisse - Novara 1549

3) Carlo Zimara et sa femme, plus Renata

Novara 1549

4) Abbé Chigi, à Turin (1549)

Après mort de Zimara (crime)

5) Naissance et mort de Robert Forestier, $1970^{22}$.

La structure alternée de l'Antiphonaire, si complexe qu'elle soit, résulte de la simplification des structures infiniment plus compliquées qui avaient été rêvées au moment du travail sur le plan. Plus précisément, elle est le produit de l'intégration de structures au départ distinctes dans une forme supérieure qui les articule les unes aux autres, tisse de ces fils qu'elle entrecroise la trame d'un récit unitaire en dépit de la diversité des éléments qui le composent. Enfin, le "Plan partiel..." comporte des «remarques formelles " qui éclairent singulièrement certaines ambiguïtés de la version finale du texte en précisant les limites qu'il convient d'accorder au principe d'alternance des récits. Sa mise en cuvre n'a rien de mécanique et la confusion de plusieurs chapitres se présente comme un antidote aux automatismes d'une trop systématique régularité:

REMARQUES FORMELLES EN CHEMIN :

- ne pas rendre les niveaux historiques trop également parallèles; - ne pas - en passant de l'un à l'autre - escamoter ainsi les scènes importantes; $[\ldots]^{23}$

Les multiples passages d'un niveau historique à l'autre et les zones d'intersection qu'ils déterminent ont certes pour fonction de les lier pour assurer l'unité du roman mais ils ne doivent pas par ailleurs abolir leur caractère distinct: Aquin ne marque pas moins leurs dissemblances que leurs analogies. II en résulte une tension qui définit la tonalité particulière de l'Antiphonaire: un seul roman engendré par la rencontre de deux récits fondus l'un à l'autre en même temps qu'ils sont distingués. Construction paradoxale dont Aquin nous livre sans doute le chiffre dans cet «Axiome de Marie la Copte" qu'il cite en épigraphe à son livre:

L'un devient le deux, le deux devient le trois, et le trois retrouve l'unité dans le quatre ${ }^{24}$.

$\mathrm{Ce}$ "quatre" où se recompose mystérieusement l'unité perdue n'est pas donné dans le roman; il définit la tâche du lecteur appelé à prolonger le 
mouvement du texte et à en résoudre les contradictions dans l'acte même de lire.

C'est que l'unité de l'Antiphonaire est marquée nettement à un autre niveau qu'à celui des grandes structures, dans le mouvement de ses phrases, dans la virtuosité affirmée d'une performance stylistique soutenue. Les deux voix s'interpénètrent en effet, se confondent dans une écriture dont les artifices assurent au roman une unité de ton très sensible même si, paradoxalement, elle se définit par le mélange continu des styles les plus hétérogènes: cette hétérogénéité constante engendre un style. Ainsi, un relevé exhaustif des noms de personne et des noms de lieu cités dans l'Antiphonaire frappe par son ampleur. Dans les 250 pages de ce roman, on ne compte pas moins de 199 noms de personne et 228 noms de lieu. Et à l'exception d'une douzaine de noms de personne ${ }^{25}$ et d'une autre douzaine de noms de lieu ${ }^{26}$ qui reviennent constamment, justifiés par le déploiement de la narration, leur occurrence reste très faible ( 6 fois et moins); 148 noms de personne et 155 noms de lieu (soit près des trois quarts de chaque liste) n'apparaissent même qu'une seule fois. On peut y voir la manifestation d'un plaisir de citer pour le plaisir de citer ou d'une fascination pour les listes et les catálogues ${ }^{27}$ puisque ces noms qui n'apparaissent qu'une seule fois, ces apaxs pourrait-on dire, paraissent gratuits dans une perspective fonctionnelle, puisqu'ils viennent gonfler anarchiquement la somme des informations que le lecteur doit maîtriser et qu'ainsi ils viennent perturber le processus de déchiffrement du texte par une sorte de brouillage, par un bruit continu de parasites. On les rencontre de place en place comme des corps étrangers à la trame du récit et, faute de pouvoir les identifier tous sans consulter interminablement des atlas et des dictionnaires biographiques (entreprise décevante dans la mesure où elle se révèle fastidieuse et peu utile, sinon nuisible puisqu'elle entraîne sur de fausses pistes, à l'intelligence du texte), on les reçoit comme interchangeables, équivalents les uns aux autres. Cette impression se trouve confirmée par le plaisir qu'Aquin semble éprouver à évoquer un même lieu ou un même personnage sous différents noms ou sous différentes formes de son nom, brouillant volontairement les pistes, égarant le lecteur. Ainsi, le si doux Monte Rosa colore délicatement le Mont Blanc; Philippe Aurèle Théophraste Bombast von Hohenheim masque Paracelse, cette vieille connaissance; Abu'l Walid Mohammed Ibn Ahmed Ibn Rosd se confond avec le très familier Averroës. Comme d'autres multiplient qui les adjectifs, qui les subordonnées, Aquin prodigue les noms propres plus pour s'enivrer de leur musique, plus pour inscrire dans la trame de son texte l'impensé que leur méconnaissance propose, que pour évoquer les personnages estimables ou médiocres, les lieux remarquables ou nuls qu'ils désignent. Ce goût des énumérations, Aquin l'a manifesté dès Prochain Épisode ${ }^{28}$ et on peut le considérer comme un trait constant de son écriture; toute étude d'ensemble de son œuvre devra en proposer une interprétation. Dans l'Antiphonaire, elles tendent à se multiplier sous divers prétextes: libreassociation du monologue intérieur (p. 17-18), évocation d'un passé heureux aux moindres détails duquel on s'accroche éperduement (p. 73), reproduc- 
tion pure et simple d'une liste préexistante (p. 105), reconstitution d'un emploi du temps comme au cours d'une enquête policière (p. 115), accumulation de preuves par citation d'auteurs dans une thèse de doctorat (p. 127-128), inventaire d'objets à reclasser (p. 138-139), reproduction du désordre fébrile d'un esprit en proie au délire (p. 209)... Aucune explication particulière, si satisfaisante qu'elle paraisse au premier abord pour tel ou tel exemple, ne saurait vraiment rendre compte de leur prolifération étonnante. C'est le retour constant du même procédé dans les contextes les plus variés qui fait question plutôt que tel de ses emplois. Sous le nom de conglobation, les anciens rhéteurs décrivent ce procédé comme une figure par emphase ou par exubérance où le langage prend une espèce d'autonomie, se met à germer, bourgeonner, proliférer pour son propre compte ${ }^{29}$. Dans l'Antiphonaire, ces excroissances linguistiques traversent la frontière des deux récits et tendent à la gommer en superposant aux différences qui les séparent (époques, rythmes, personnages, événements, point de vue de narration) une couleur stylistique relativement constante. Cette couleur plus ou moins uniforme a pour effet de mettre au premier plan, au dessus de la trame de la narration, une surface scripturale où le travail de production est patent. Les énumérations viennent ainsi rompre la succession des événements et arracher à ses phantasmes le lecteur occupé à «suivre l'histoire». Elles lui rappellent ironiquement qu'il lit, que les représentations dont il est la scène résultent de l'activité de son esprit, et qu'il se trouve confronté à des artifices. L'illusion réaliste nécessaire à la lecture d'un roman (puisque lorsque je lis je dois m'abstraire de cette chambre où je suis assis, tenant ce livre dans mes mains, tournant les pages, oublier ce lieu où je me trouve, cesser de voir ce paysage qui se déploie à la fenêtre, quitter tout cela qui est présent pour entrer dans une absence paradoxalement plus prégnante que ce qui m'entoure, pour vivre dans une distance équivoque des aventures terribles ou délicieuses même si elles ne sont faites que de mots ${ }^{30}$ ) s'en trouve temporairement ébranlée et doit être reconstituée au prix d'un nouvel effort d'imagination. Dans ces conditions, lire cesse d'être un abandon plus ou moins passif aux pouvoirs d'un texte qui prendrait en charge la rêverie de celui qui s'y plonge; le lecteur doit collaborer au texte, l'orienter à chaque page, et il ne lui confère de sens qu'au prix d'un investissement imaginaire constamment renouvelé.

Ces énumérations lyriques qui traversent la coupure des deux niveaux du roman transforment en profondeur la narration en lui superposant une structure poétique de type itératif. Ce n'est plus exclusivement, ni peutêtre même d'abord, la succession des événements qui fait progresser la lecture; celle-ci se trouve informée par le retour périodique d'une structure qui offre sa présence immédiate, hors de la visée représentative, à la manière d'un refrain ou d'un «thème» insistant dans un long poème. La composition de l'Antiphonaire, par conséquent, s'avère régie par une structure aussi serrée que celle d'une grande ode malherbienne. Les intenses ncuds verbaux des énumérations y jouent un rôle analogue à celui des rimes dans la poésie classique, ils scandent le texte et superposent à la progression linéaire de la narration une architecture nythmique, l'espace d'une poly- 
phonie. Paradoxalement Aquin, qui s'est toujours obstinément refusé à écrire. des poèmes ${ }^{31}$, reprend ainsi en le projetant dans le roman un des procédés lyriques les plus fondamentaux, l'un de ceux notamment qui définissent le mieux aujourd'hui le poétique depuis l'abandon de la prosodie classique. Ce paradoxe se dissipe toutefois dans la mesure où, comme l'a montré Michel Butor, tout roman d'un certain niveau fait appel à une “prosodie généralisée ${ }^{32}$ ". Aussi l'édifice qu'élabore le retour des énumérations a-t-il pour fonction de signaler une autre prosodie plus secrète: l'ordre des actions et des événements.

\section{Cinéma verbal}

Ceux-ci ne se réduisent pas en effet au prétexte qui servirait de support aux voix alternées de la narration et au lyrisme des énumérations. Les événements, dont la succession ordonnée (quel que soit le principe qui gouverne cette succession) constitue la trame de tout récit, se déploient dans l'Antiphonaire selon un désordre apparent qui camoufle une disposition en fait aussi rigoureuse que celles que décrivent les anciennes rhétoriques. Comme les épopées classiques, et selon les recommandations des anciens rhéteurs, l'Antiphonaire commence "in media rerum", au milieu de l'histoire plutôt qu'à son début; le récit s'en trouve orienté d'emblée, transformé en argument. Une série de retours en arrière, qui superpose à la progression des épisodes vers leur résolution future des mouvements en sens inverse vers leur genèse, devient nécessaire. Façon toute classique de procéder puisqu'un traité comme l'Abbrégé de l'Art poëtique françois de Ronsard, par exemple, ne recommandait pas, en 1565, de procéder autrement ${ }^{33}$. Ce n'est pas là qu'il faut chercher la modernité de l'Antiphonaire. Par contre, la fragmentation systématique de la suite narrative conduit à un traitement très neuf de la matière romanesque selon des techniques plus ou moins analogues à celles qu'utilise le cinéma: le découpage en fragments ou en "plans" distincts et leur montage en séquences de dimensions variables qui contrastent violemment entre elles ou, plus rarement, s'enchaînent sans la moindre dissonance ${ }^{34}$.

Avant d'entreprendre l'analyse du montage dans l'Antiphonaire, il faut toutefois réfuter une objection fréquente. L'utilisation littéraire de techniques empruntées à un autre art impose des transformations telles qu'on peut difficilement ne pas les considérer comme des techniques nouvelles, et il ne va pas de soi, par conséquent, qu'une analyse littéraire qui empruntera ses concepts à la théorie du montage cinématographique ne déformera pas son objet et ne se réduira pas à l'emploi purement métaphorique, sans valeur démonstrative, d'un vocabulaire ${ }^{35}$. En guise de réponse, on pourrait être tenté de citer purement et simplement l'étonnante scène de l'Antiphonaire (p. 210-211) où l'utilisation du langage technique du cinéma évoque des procédés de composition susceptibles, à première vue, d'éclairer l'ensemble du livre; mais cette dépense métaphorique annonce plus, presque sur un mode à l'avance parodique, le découpage scénique généralisé de Neige noire qu'elle ne désigne les techniques de montage mises 
en cuvre dans l'Antiphonaire. Par contre, le "Plan partiel de l'Antiphonaire " dans lequel Aquin parle à plusieurs reprises de "fondu enchaîné ${ }^{36}$ " indique de façon précise à quelles techniques il a eu recours et montre qu'il ne s'agit pas d'une simple analogie commode permettant d'escamoter l'analyse. Enfin, comme Eisenstein l'a amplement prouvé, le montage cinématographique n'est qu'un cas particulier d'une technique générale de montage qui intéresse tous les arts, aussi bien la littérature et la peinture que le cinéma ${ }^{37}$.

Dans l'Antiphonaire, les traces de montage apparaissent avec le plus d'évidence aux moments où le texte bifurque d'un niveau historique à l'autre. Au troisième chapitre, par exemple, l'année 1969 devient brusquement l'année 1536 dans un étonnant télescopage chronologique. Christine Forestier se métamorphose en Renata Belmissieri grâce à l'ambiguité sémantique du pronom personnel de la première personne du singulier, sorte de pince linguistique qui replie brutalement les époques l'une sur l'autre:

Je ne tourne pas quand je tombe, mais - oh, boy... - je tombe bêtement sans prévenir, sur les carreaux, dans les escaliers, à San Diego et même sur le chemin vicinal entre Novara et Chivasso, ville absolue (cita absoluta) vers laquelle je me dirige, pas à pas, porteuse artisanale d'un manuscrit précieux dont j'ai pris livraison à la frontière suisse et qu'un imprimeur de Chivasso attend avec angoisse pour l'imprimer (il s'agit du manuscrit du «Traité des maladies nouvelles " de Jules-César Beausang). Mon nom est Renata Belmissieri, italienne de naissance, née à Cremona, battue par ses parents, blessée par la vie et trouvant quelque argent dans la translation de manuscrits interdits par la censure royale de France... Je vis de cette étrange contrebande. (p. 28)

Ce procédé peut être défini comme la projection dans la chaîne syntaxique du mécanisme instantané d'une métaphore. Celle-ci rapproche en effet deux termes distincts par le biais d'une analogie, selon tous les rhéteurs depuis Aristote ${ }^{38}$ (la nouveauté apportée par Reverdy et Breton, devenue caractéristique de toute la modernité, est la distance aussi grande que possible qui doit séparer les deux termes ${ }^{39}$ ). Les auteurs de la récente Rhétorique générale ont précisé le fonctionnement linguistique de ce principe d'analogie et redéfini la métaphore comme le passage d'une relation d'intersection entre les deux termes («partie commune à la mosaïque de leurs sèmes ou de leurs parties") à leur réunion complète (“identité des termes entiers") $)^{40}$. Dans le texte d'Aquin, ce passage (instantané dans la poésie lyrique où on laisse au lecteur le soin d'en reconstituer - ou de tenter de le faire - les étapes intermédiaires) s'effectue progressivement, dans le déroulement même de la phrase où les premiers «je», étant donné le contexte, désignent Christine, les suivants, troubles et comme dérapant, à la fois Christine et cette Renata Belmissieri qui n'a pas encore fait son apparition ou plutôt un être brouillé, flou, incertain, qui n'est ni l'une ni l'autre, et les derniers, la seule Renata Belmissieri qui évacue Christine grâce à un luxe de circonstances, presque une fiche biographique qui signale l'entrée en scène d'un nouveau personnage. Ailleurs, ce sera un objet qui assurera le passage d'un terme à l'autre en changeant de statut. 
Par exemple, le chapitre 11 (p. 83) s'ouvre par des considérations d'histoire des idées, ostensiblement savantes, sur la pensée médiévale, qui offrent l'occasion d'énumérer les titres de nombreux ouvrages de cette époque; le lecteur se trouve en quelque sorte placé devant un fragment de la thèse de doctorat que prépare Christine Forestier. Puis il se déplace brusquement au $X V I^{\mathbf{e}}$ siècle après que son attention ait été fixée sur un. ouvrage particulier qui lui est aussitôt montré dans les mains d'un personnage; au lieu d'un commentaire sur le contenu de ce livre comme ceux qui viennent d'être esquissés à propos d'autres livres, c'est un abbé lisant dans un jardin, en 1536, qui apparaît:

Le «Theatrum chemicum» publié vers le début de la Renaissance (bien qu'anonyme) connut un succès foudroyant - à l'image justement de ce Moyen Âge diffusif et dilaté. En ce 10 août 1536, à Turin, l'abbé Leonico Chigi lisait le «Theatrum chemicum», tout en arpentant le petit jardin qui formait la cour intérieure du presbytere de l'église paroissiale de San Tomaso. (p. 84)

Le mécanisme est le même, à cette différence près que le passage ne s'effectue plus dans la texture syntaxique même mais au niveau de représentations qui changent brusquement de statut: le "Theatrum chemicum ", la première fois, désigne un texte, un type de pensée caractéristique d'une époque; il devient ensuite un livre, un objet matériel d'un format qui permet de le tenir dans les mains en se promenant dans un jardin.

Ces passages - les « fondus enchaînés" du «Plan partiel... — - qui réorientent continuellement les différentes séquences de l'Antiphonaire confèrent au récit une allure sinueuse, marquée de contrastes et de ruptures: il progresse par des réajustements successifs, d'incessants déplacements de point de vue. Le sens de chaque séquence se charge d'incertitude dans la mesure où les éléments qui les composent entreront, à l'intérieur des séquences subséquentes, dans de nouveaux réseaux de relations qui modifieront radicalement la perception qu'on peut en avoir. Le lecteur devient la proie d'un soupçon qui double la foi (suspension volontaire de l'incrédulité pour un moment, disait Coleridge) dont la lecture se soutient d'une sorte d'ombre critique où la mémoire de significations antérieures et l'appréhension de significations à venir contradictoires jettent le trouble parmi celles qui se déploient, hic et nunc, sur la page. Plusieurs interprétations concurrentes se révèlent possibles à chaque moment, et le lecteur, sollicité dans plusieurs directions à la fois, doit résoudre sans cesse des conflits qui lui sont imposés. L'effet d'un tel mode de composition a été remarquablement décrit par Eisenstein selon qui "la juxtaposition de deux fragments [...] ressemble plus à leur produit qu'à leur somme [...] en ce que le résultat de la juxtaposition diffère toujours qualitativement (calculé, si l'on préfère, en exposants) de chacune des composantes prises à part ${ }^{41}$ ": “les représentations se combinent en images ${ }^{42}$ " produites par l'activité du lecteur à partir des éléments qui sont fournis. C'est lui qui construit la totalité sans cesse remise en question en organisant les fragments qui lui sont proposés. Et dans l'Antiphonaire, Christine, en commentant le style de l'autobiographie de Leonico Chigi, définit en fait le mode de 
composition de tout le roman et ce qui lui semble sa portée, en sorte que cette machine à lire contient son propre mode d'emploi :

Cette technique qui lui venait peut-être de la lecture quotidienne de son bréviaire (soit: un chapitre par jour) ressemblait à une technique de la fragmentation; ainsi, Chigi composait son récit autobiographique en plusieurs fragments (ou tableaux) mal reliés les uns aux autres, disloqués, disjoints. L'ensemble donne une forte impression de discontinuité, de découpage brutal! Rien n'est plus étrange que cette manière discordante qui brise encore plus qu'elle ne compose et qui défait, au fur et à mesure, le peu qui a été assemblé. $A$ mes yeux, ce procédé n'a rien de privatif ou d'inefficace dans la confection d'un livre quel qu'en soit le sujet; il pourrait tout aussi bien s'agir d'un traité scientifique que d'un livre de fiction, la lecture n'en serait pas plus difficile parce que l'auteur aurait utilisé cette méthode de fragmentation. Du moins, c'est ce que je crois bien naïvement. Peut-être suis-je en train de projeter sur la méthode employée par Chigi parce que sa vision atomisée de la réalité et la façon qu'il a de la représenter me conviennent personnellement? Rien d'autre. (p. 217-218)

\section{Apologie de l'artifice}

Une lecture naïvement réaliste de l'Antiphonaire se révèle donc pratiquement impossible: le texte la déçoit sans cesse, détruisant avant qu'elle ne parvienne à prendre corps toute représentation cohérente qui prétendrait se substituer à lui. Le lecteur peut difficilement s'abandonner au rêve éveillé qui consiste à "suivre l'histoire" parce qu'il ne peut oublier qu'il lit un roman et que la fiction ne lui est pas «donnée», qu'il doit l'élaborer lui-même à partir des signes qu'il déchiffre sur la page. Cette fiction, Aquin en souligne à plusieurs reprises le caractère aléatoire et lui retire du même coup toute valeur exemplaire, toute nécessité qui justifierait sur un mode transcendant la succession de ses péripéties. Au lieu du cosmos d'une représentation tragique, c'est une surface qui se déplie devant nous, et les événements qui y prennent place se réduisent à des rencontres fortuites, proposent une trame narrative incertaine, qui pourrait être autre, qui se vide de réalité par l'invocation d'autres trames restées virtuelles, purs possibles qui auraient pu la remplacer:

Les improbabilités, j'imagine, sont corrélatives aux probabilités, et, dès lors, on peut déduire que l'improbabilité ou la probabilité de manifestations épileptiques agressives sont l'une et l'autre possibles. Ce genre de raisonnement se pratique, à ce que j'en sais, en mathématiques: le probable et l'improbable sont assimilés à des possibles tout simplement. Or, rien n'est à proprement parler impossible... Par exemple, je pouvais, en manœuvrant la Cutlass dans les rues de San Diego, après la mort du pharmacien... j'aurais pu - admettons frapper mortellement un piéton qui n'aurait été autre que mon propre mari : Jean-William... ou encore: un des enfants du pharmacien. Mais rien de tout cela n'est arrivé; c'étaient des possibilités, elles sont demeurées telles... (p. 103)

Très souvent dans l'Antiphonaire, trop pour que cela ne soit pas calculé, la mise en relief des artifices vient contester l'illusion réaliste. Aquin va rappeler, par exemple, de façon inattendue et incongrue que les représentations tirent leur origine de l'acte d'écrire dans ce qu'il a de plus maté- 
riel - l'inscription patiente de signes de gauche à droite sur les lignes dont l'empilement dessine le bloc typographique de la page:

Il est temps que Chigi, qui déambule le long de ces lignes et sur les quais de Genève, arrive enfin à l'appartement qu'il occupe avec sa compagne Antonella. (p. 175-176)

Ailleurs il introduit une distance critique au cœur même du récit en élaborant des commentaires stylistiques ostensiblement compliqués, parodiques presque, où les rapports de l'écriture et de la fiction qu'elle porte se mettent à faire question:

Je m'abandonne à l' "ornata facilitas» (nimis duris et ampullosis translationibus ", aurait dit Geoffroi de Vinsauf...). L' ' ornata facilitas "; ce n'est rien encore, quand on pense à la "stilorum incongrua variatio" ou aux "vitia colletaria", tels que décrits par Jean de Garlande. Non, je n'ai pas perdu pied encore dans cette mer sombre et salée ("stilo humili...») sur laquelle je tente d'effectuer un naufrage vicié par le mélange des styles. (p. 203-204) (44)

Les personnages, auxquels on pourrait être tenté de se raccrocher pour édifier une représentation, n'échappent pas non plus à cette incertitude. Ainsi en va-t-il de Christine: le texte dont elle est censée être l'auteur est parsemé d'invocations aux lecteurs, invraisemblables parce que le journal qu'elle tient ne peut pas être destiné à la publication; de cette façon, son rôle de personnage témoin s'efface et elle prend la pose d'un écrivain professionnel qui s'adresse à un public familier (p. 20, 69, 105,...). Enfin, Aquin varie systématiquement les styles et les registres d'écriture de manière à mettre en évidence leur caractère artificiel, à souligner par une virtuosité ostentatoire qu'ils sont le produit d'une élaboration plutôt que le fruit spontané d'une inspiration ou la transcription sans apprêts d'un témoignage: style “série noire", d'autant plus voyant qu'il décrit des événements du XVI ${ }^{\ominus}$ siècle (p. 91-93), styles de roman "gothique" (p. 169), de roman « médical " (p. 176), de roman sentimental (p. 232), etc. IIs occupent les lieux les plus inattendus du récit durant quelques lignes ou quelques pages qui évoquent des domaines littéraires extrêmement variés - de ce qui est généralement tenu pour la "grande" littérature jusqu'aux différents sous-genres de la littérature «de masse».

Cette mise en évidence de l'artifice ne détruit évidemment pas tout à fait l'illusion réaliste, ce qui rendrait impossible la lecture; mais elle l'ébranle assez pour qu'on ne puisse pas s'y abandonner en toute innocence. Aquin a évoqué les conséquences de cette attitude dans un texte théorique écrit au moment où il préparait l'Antiphonaire ${ }^{45}$ et qui, pour cette raison, est susceptible d'en éclairer la lecture:

II me semble impérieux de désacraliser l'inspiration inconsciente comme détenant une position privilégiée; mais, je ne crois pas, pour autant, qu'il faille démythifier la production littéraire afin de rejoindre un au-delà de l'artificialité spécifique à la littérature...

Au contraire, je préconise qu'on sacralise, ni plus ni moins, cette artificialité qui, selon moi, est inhérente à tout ce qui est écrit - de telle sorte que, rendue consciente, cette artificialité révèle la propriété par excellence de produit littéraire, soit: les techniques de composition de l'écrivain... 
Ainsi perçue, la littérature nous apparaît comme un échange entre le lecteur et l'écrivain - échange qui correspond au degré d'implication et de compréhension du lecteur...46

L'ostentation rhétorique qui parcourt l'Antiphonaire apparaît donc comme la mise en œuvre d'une esthétique. L'ornement (au sens que les anciens rhéteurs donnaient à ce mot) y devient une valeur en soi et les figures se mettent à proliférer pour leur propre compte, hors de toute visée fonctionnelle (dans un discours, persuader l'auditeur; dans un roman, convaincre le lecteur, au moins pour la durée de la lecture, que ce qu'il lit est vraisemblable, donc, en quelque façon, vrai): ainsi, certains événements se trouvent repris, racontés plus d'une fois selon des points de vue différents, non pour offrir au lecteur la possibilité de construire une vision totalisante par l'accumulation de diverses approches partielles d'un même objet qui préexisterait à l'acte d'écrire, mais pour élaborer des variations qui proposent un objet identique et différent à la fois, dont la structure abstraite et la succession harmonique se suffisent à elles-mêmes. Les réflexions sur l'écriture qui interrompent la narration et qui agaceraient un lecteur pressé de "savoir la suite" participent du même esprit. Elles n'éclairent pas les événements, ne projettent sur eux aucune signification, parce que la hiérarchie courante entre le contenu de la narration et son mode de fonctionnement n'a plus cours: l'enchaînement des péripéties n'a pas plus d'importance que les variations stylistiques dont elles font l'objet, et les commentaires techniques intégrés au texte se déploient comme autant d'explorations de nouvelles zones de langage. L'œuvre devient décentrée (la notion même d'œuvre est remise en question), elle se morcelle indéfiniment, elle se multiplie en fragments où le lecteur va de surprise en surprise, incapable de trouver un point à partir duquel il serait possible d'unifier cet archipel de parties. L'illusion se donne pour telle, l'écrivain faisant étalage des procédés, techniques, ficelles qui lui permettent de la faire naître et de l'imposer. À l'adhésion à la fiction qui est la condition première de la lecture d'un roman se superpose ainsi une distance critique qui en déplace sans cesse les perspectives ${ }^{47}$.

\section{Histoire de la mesure du temps}

Le roman devient alors un univers régi par d'autres lois que celles de la représentation vraisemblable. Aussi le temps prend-il dans l'Antiphonaire des formes étonnantes, profondément différentes de celles qui gouvernent le monde physique ou simplement la vie quotidienne. Bien sûr, "le temps, irréparable selon Horace, fuit" (p. 21) et "les jours passent" (p. 121) dans ces pages un peu comme dans la vie de chacun. Mais ce n'est là qu'une des modalités de cet «espace-temps " infiniment complexe et malléable que Christine décrit dès l'ouverture de son récit, dans la distance étrange d'un détachement presque serein, comme si tout avait eu lieu avant même d'avoir commencé:

Je me meus sans émotion dans un espace-temps dont les frontières sont difficiles à discerner: bien sûr, il y a d'abord ce lit du motel Hillcrest à San Diego, mais je suis encline à ne plus m'y voir auprès 
de Jean-William qui continue son épisode épileptiforme sans moi, puis Montréal sort de la brume (qu'on appelle "smog "à San Diego), Montréal où j'ai vécu trop longtemps et trop mal, puis Bâle où JulesCésar Beausang, disciple de Paracelse, vécut en exil, puis Turin, puis Chivasso, sur les bords de la Sesia, ancien village devenu villesatellite du grand Turin... (p. 17)

Les événements de 1969 devraient vraisemblablement être postérieurs à ceux de 1536. Pourtant le lecteur découvre que les durées à l'intérieur desquelles ils se produisent ne se succèdent pas mais qu'elles se superposent comme les différentes «parties » sur du papier à musique, qu'elles sont parallèles, simultanées:

Mais tandis qu'il (l'abbé Chigi qui voyage de Genève à Lyon en 1537) se repose je ne sais où entre Bourg-en-Bresse et Lyon, moi - Christine - je ne sais plus quoi penser des renseignements contradictoires que je reçois au sujet de Robert. (p. 192-193)

Les représentations que suggère le roman (et qui peuvent se concrétiser, par exemple, dans la forme d'un résumé) restent, bien sûr, conformes à une certaine vraisemblance et les événements de 1536 y sont antérieurs à ceux de 1969: comment d'ailleurs pourrait-on se représenter ou penser un temps autre, à moins d'accepter les conventions du genre fantastique? C'est dans le texte d'Aquin qu'il en va autrement, qu'une autre durée parvient à se déployer. Le temps de l'Antiphonaire est un temps écrit: il n'a d'existence que par les marques linguistiques qui le signifient et il est par conséquent susceptible de toutes les manipulations et de toutes les transformations que permet la morphologie des verbes français. On assiste ainsi à la transformation de Christine en Renata Belmissieri grâce au passage d'un présent contemporain de l'écriture (qui coïncide avec l'inscription des mots sur la page) au passé simple d'un récit objectif à la troisième personne (qui se situe ostensiblement après les événements révolus qu'il narre, dans la distance de l'accompli et du neutre):

Pauvre Renata Belmissieri, mon double, cette jeune fille qui me sert de personnage-victime quand je tente, par projection, d'imaginer et de figurer une femme en proie aux spasmes récurrents de la crise d'épilepsie. Elle a pris livraison d'un manuscrit précieux; et, pour ce travail, elle est bien payée. Mais c'est de la contrebande; donc le risque est grand. Elle a pris son colis à San Bernardino, tout près de la frontiere suisse. Elle sait qu'elle doit le porter à un imprimeur qui se nomme Carlo Zimara, Calle Santa Clara, 6, Chivasso. Elle connaît le chemin de San Bernardino à Novara, puis de Novara à Chivasso, sur les bords de la Sesia. [...] Elle est partie à l'aube de San Bernardino en cachant le manuscrit sous sa jupe; puis, lasse de marcher lentement à cause de cet obstacle, elle a déposé le manuscrit sous une masse d'épices qu'elle a transporté avec plus d'aisance et d'un pas plus soutenu. De toute façon, elle savait qu'elle n'atteindrait pas Chivasso avant la nuit.

Elle trouva, vers l'heure du crépuscule, un bosquet tranquille en retrait de la route de Turin... (p. 29-30)

Ce temps produit par l'écriture, les artifices littéraires permettent d'en changer le cours, de le remonter, le rattrapper, le retrouver, l'accélérer, le ralentir, le varier indéfiniment. Comme tel, il échappe aux lois du temps phy- 
sique où le futur ne cesse de se déverser dans le passé à travers un présent essentiellement transitoire; il en diffère autant que l'espace d'un tableau diffère de l'espace physique réel. Pour bien marquer cette rupture fondamentale, Aquin a encadré la fiction, lieu de cet espace-temps aux propriétés déconcertantes, de chapitres qui reproduisent de façon vraisemblable le temps réel. L'Antiphonaire est ouvert par un chapitre de style réaliste (le réalisme est un style) où un narrateur objectif, détaché de la fiction à laquelle il demeure étranger, compose une espèce d'introduction au récit proprement dit. Celui-ci ne commence vraiment qu'à partir du deuxième chapitre, au moment où c'est Christine qui écrit:

Ici débute le livre que j'ai constitué à même les documents et les diverses pièces de ce dossier. (p. 17)

Et à la fin, une Postface due à Suzanne Bernatchez et une lettre d'adieu d'Albert Franconi, l'un et l'autre personnages relativement épisodiques, referment le roman. Cette introduction et cette double conclusion tracent les limites du temps de la fiction, entourent (enferment) le langage délirant auquel s'abandonne Christine, à la façon d'un cadre qui sépare un tableau du mur sur lequel il est accroché en même temps qu'il souligne sa présence.

Pour marquer que son roman joue du temps, Aquin lui a donné un autre titre, très visible, offert sans détour, mais pourtant secret et perçu sans doute par peu de lecteurs: les deux gravures reproduites sur la couverture $^{48}$. Elles sont identifiées en ces termes:

Sur la couverture: / Gravures sur cuivre de F. Berthoud, / dans «Histoire de la mesure du temps", / Paris 1802, / figurant le rouage d'une horloge à poids / avec echappement à verge et foliot, / puis une coupe transversale / du mouvement à verge d'une montre de poche. (p. 4)

L'Antiphonaire, chant alternatif, est aussi une «histoire de la mesure du temps", une machine double - il y a deux gravures sur la couverture aux rouages précis et compliqués, dont nous avons à découvrir les mécanismes, les rythmes, les lois, que nous avons à faire fonctionner.

Faut-if en conclure que ce roman constitue une monade littéraire, qu'il n'entretient aucune relation avec la réalité et qu'il se trouve de ce fait dépourvu de sens: un bibelot pervers et compliqué, parfaitement gratuit, dans la contemplation duquel il est loisible de s'absorber mais auquel on ne saurait accorder aucune portée? Avec trois meurtres et deux tentatives de meurtre, trois suicides, une demi-douzaine de crises d'épilepsie, quatre ou cinq viols; avec ces frénétiques voyages et cet incessant chassé-croisé de couples qui ferait pâlir un auteur de boulevard (Christine quitte Jean-William pour vivre avec Robert qui abandonne Suzanne, laquelle va vivre avec Albert Franconi qui la trompe avec Christine qui trompe Robert): avec d'innombrables invraisemblances ${ }^{49}$ - I'Antiphonaire n'est-il qu'une histoire à dormir debout? De toute évidence, il déçoit le liseur de romans, curieux de l'intrigue et de ses péripéties palpitantes, qui s'y aventure au risque de n'y voir qu'un mauvais mélodrame ou un livre 
bâclé50. Mais cette déception ne résulte pas de maladresses, Aquin l'a délibérément provoquée après l'avoir préméditée:

- A la fin, la composition doit se détraquer: une sorte de contagion frissonnante fait se fondre, avec confusion et désordre, des intrigues distinctes nettement et éloignées l'une de l'autre dans le temps: [...] Et, si possible, tout finir en queue de poisson... ${ }^{51}$

II coupe court de cette façon à la possibilité même d'une interprétation naïvement globalisante qui trouverait à se justifier, malgré les contradictions du texte, dans quelque sens «final". Ce texte impossible a évacuer, à remplacer par des significations traduisibles dans un autre discours, ne peut faire l'objet que d'une performance de lecture, toujours inachevée, qui le fasse fonctionner.

Son titre, qui évoque le rituel (un “antiphonaire» est un livre d'église où les antiennes et autres chants de l'office sont notés en caractère de plain-chant), peut indiquer qu'il nous convie à une "célébration ${ }^{52}$ ": II serait, a-t-on écrit, une "anti-messe qui a sa propre liturgie, sa propre antienne (celle de l'échec), ses saints et son dieu, le $\mathrm{Mal}^{53}$ ". Rite en quelque sorte de la mort de Dieu et peut-être invocation de l'inimaginable mauvais démiurge. Mais plus sans doute que les outrances d'une somptueuse et vaine messe noire, l'Antiphonaire, par les moyens paradoxaux de la mystification faussement érudite, de la parodie et de la déception stylistique savamment cultivée, propose le procès-verbal du naufrage de l'ordre et des savoirs auquel est en proie aujourd'hui l'Occident. Et la scène bouleversante dans laquelle Christine s'éveille à l'aube devant un téléviseur vidé de toute image:

Ce soir-là (celui de mon retour par Chicago à Montréal) je m'endormis toute habillée sur le lit alors que je regardais une émission policière au Canal 12: je me réveillai a l'aube, surprise, devant une petite image vide qui émettait un grésillement égal et assez faible. (p. 113)

pourrait servir d'emblême à ce roman de la destruction qui force son lecteur à s'éveiller dans cette aube vide où tout est à recommencer.

Robert Mélançon

Université de Montréal

1. "Hubert Aquin et le jeu de l'écriture", entretien avec Anne Gagnon, Voix et images, vol. I, $n^{\circ} 1$, septembre 1975, p. 8.

2. Idem, p. 8.

3. Idem, p. 9: «[...] je me réjouis de lui rendre la lecture difficile. Je me suis même réjoui de ce que certains lecteurs aient décroché de Trou de mémoire tellement ils étaient abasourdis ou ahuris."

4. Hubert Aquin, Neige noire, Montréal, Éditions La Presse, 1974, “prière d'insérer", p. 4 de la couverture. 
5. Hubert Aquin, «Quelle part doit-on réserver à la littérature québécoise dans l'enseignement de la littérature? " , Liberté, $n^{\circ} 57$ (vol. 10, $n^{\circ} 3$ ), mai-juin 1968, p. 75.

6. Hubert Aquin, "la Disparition élocutoire du poète $*, C u /-Q, n^{\circ} 4-5$, été-automne 1974, p. 9.

7. "Hubert Aquin et le jeu de l'écriture», op. cit., p. 10.

8. Idem, p. 6: “Je ne vois pas pourquoi je serais mandaté pour exprimer cette vérité ontologique par des livres ou dans des livres. "

9. Hubert Aquin, "la Disparition élocutoire du poète", op. cit., p. 6.

10. Ct. Roland Bourneuf, "Un procédé narratif: les récits alternés", Revue canadienne de littérature comparée, vol. II, $\mathrm{n}^{\circ} 2$, printemps 1975, p. 130: “... une rupture de ton, de rythme, d'action qui oblige le lecteur à des réajustements renouvelés".

11. Cf. Jorge-Luis Borges, "l'Art narratif et la magie», Discussion, Paris, Gallimard, 1966, p. 66-80.

12. Albert Chesneau, "Déchiffrons l'Antiphonaire", Voix et images, vol. $1, \mathrm{n}^{\circ} 1$, septembre 1975, p. 32.

13. Idem, p. 32.

14. Albert Léonard, "Un romancier virtuose: Hubert Aquin. A propos de l'Antiphonaire", I'CEuvre litteraire et ses significations, Montréal, PUQ, 1970. Repris dans Réal Ouellet (éd.), les Critiques de notre temps et le Nouveau roman, Paris, Garnier, 1972, p. 164.

15. Michael Beausang, "Music and medicine $"$, Canadian Literature, $n^{\circ} 58$, automne 1973, p. 73: "Antiphony is literally the art of "counter-sounding", of playing off one choral group against another. This is, of course, exactly what Aquin does in L'Antiphonaire, where modern and Renaissance choral groups counterpoint one another across an acoustical gap of well over four hundred years. "

16. Roland Bourneuf, "Un procédé narratif: les récits alternés ", op. cit., p. 137.

17. Hubert Aquin, Points de fuite, Montréal, Le Cercle du livre de France, 1971, p. 91-111. Aquin a dit récemment toute l'importance qu'il attache au « travail de conception» qui débouche sur la mise au point d'un plan où atout l'enchaînement des séquences " se met en place, et en regard duquel, à ses yeux, «l'écriture, l'exécution [...] est un tout petit peu secondaire" («Hubert Aquin et le jeu de l'écriture", op. cit., p. 6). Le "Plan partiel de l'Antiphonaire " est donc plus qu'un document susceptible d'intéresser un historien de la littérature: on peut y saisir l'étape la plus importante de la genèse du texte et suivre le déploiement progressif de ses formes.

18. Hubert Aquin, Points de fuite, op. cit., p. 97.

19. Idem, p. $98,106,107,110$.

20. «Hubert Aquin et le jeu de l'écriture», op. cit., p. 6.

21. Hubert Aquin, Points de fuite, op. cit., p. 108.

22. Idem, p. 111.

23. Idem, p. 104. C'est Aquin qui souligne.

24. L'Antiphonaire, p. 7.

25. Jules-César Beausang, Renata Belmissieri, Robert Bernatchez, Suzanne Bernatchez, Leonico Chigi, Christine Forestier, Jean-William Forestier, Albert Franconi, J.-L. Gordon, Antonella Zimara, Carlo Zimara.

26. Bâle, Chivasso, Genève, Hôpital du Sacré-Cœur, Lyon, Montréal, Motel Hillcrest, San Diego Drugstore, San Diego, San Francisco, Santa Barbara, Turin.

27. «Hubert Aquin et le jeu de l'écriture», op. cit., p. 17-18. Cette passion pour les listes doit être rapprochée de l'intérêt qu'Aquin porte “aux tracés de frontières" qu'il évoque dans le même entretien: la liste est elle-même une frontière du savoir et elle relève, elle aussi, de la fascination des limites et de l'inconnu, de l'impensable, qui se déploie au delà. Cf. Jean-François Lyotard, «la Confession coupée», Butor: Colloque de Cerisy, Paris, UGE, "10-18", 1974, p. 129.

28. Hubert Aquin, Prochain Épisode, Montréal, Le Cercle du livre de France, 1965, p. 10 et 30 , à titre d'exemples.

29. Ct. Pierre Fontanier, les Figures du discours, Paris, Flammarion, 1968, p. 363. 
30. Cf. Michel Butor, "le Roman et la poésie " et "l'Espace du roman ", Répertoire II. Paris, Éditions de Minuit, 1964, p. 7-8 et 43.

31. Jacques Folch, "Entretien avec Hubert Aquin», Liberte, $n^{\circ} 42$, (vol. $7, n^{\circ} 6$ ), novembre-décembre 1965, p. 506.

32. Michel Butor, “le Roman et la poésie», op. cit., p. 22-23.

33. Pierre de Ronsard, Abbrégé de l'Art poëtique françois, OEuvres complètes, éd. G. Cohen, Paris, Gallimard, "La Pléiade", 1951, tome II, p. 1000: "Tu doibs sçavoir sur toutes choses que les grans poëmes ne se commencent jamais par la premiere occasion du faict, ny ne sont tellement accomplis, que le lecteur espris de plaisir n'y puisse encores desirer une plus longue fin; mais les bons ouvriers le commencent par le milieu, et sçavent si bien joindre le commencement au milieu, et le milieu à la fin, que de telles pieces raportées font un corps entier et parfaict."

34. Sur la théorie du montage, cf.: V.I. Pudovkin, On film technique, London, Vision Press, 1950; S.M. Eisenstein, Film form, Essays in film theory, and the Film sense, Cleveland et New Yrok, Meridian Books, 1957; S.M. Eisenstein, Réflexions d'un cinéaste, Moscou, Editions du Progrès, 1958; Change, $n^{\circ} 1$, "le Montagen, Paris, 1968.

35. Cf. Jean Ricardou, Problèmes du nouveau roman, Paris, Seuil, 1967, p. 69-88.

36. Hubert Aquin, "Plan partiel de l'Antiphonaire ", op. cit., p. 97-98, 107, 108.

37. Cf., à titre d'exemple, l'analyse d'un tableau de Léonard de Vinci dans S.M. Eisenstein, Réflexions d'un cinéaste, op. cit., p. 87-91.

38. Aristote, Poétique, $1457 \mathrm{~b}$.

39. André Breton, Manifestes du surréalisme, Paris, Gallimard, “Idées", 1963, p. 29-33.

40. J. Dubois, F. Edeline et alii (Groupe " $\mu »)$, Rhétorique générale, Paris, Larousse, 1970, p. 106-109.

41. S.M. Eisenstein, Réflexions d'un cinéaste, op. cit., p. 75. C'est Eisenstein qui souligne.

42. Idem, p. 84. C'est Eisenstein qui souligne.

43. Idem, p. 92.

44. Cf. entre autres, p. 115, un passage où le temps dérape littéralement.

45. L'Antiphonaire a été écrit durant l'été 1969. Le "Plan partiel de l'Antiphonaire ", qui constitue la première élaboration encore tâtonnante de l'argument du roman, est daté de mai 1969. Par ailleurs, dans une page en date du 9 septembre 1969 (Points de fuite, oo. cit., p. 113), Aquin indique qu'il a "terminé L'Antiphonaire il $y$ a tout juste 8 jours" et que "le texte (dactylographié) est au C.L.F." où il sera publié au cours de l'automne - l'achevé d'imprimé du premier tirage porte la date du 24 novembre 1969. Le roman a donc été écrit assez rapidement, en à peine quatre mois si l'on tient compte de la préparation du plan.

46. Hubert Aquin, "la Mort de l'écrivain maudit", Liberté, $n^{\circ} 63-64$ (vol. 11, $n^{\circ} 3-4$ ), mai-juillet 1969 , p. 26-27.

47. On peut être tenté d'invoquer la notion de baroque, mais ce serait risquer de masquer le texte (de l'évacuer) sous une grille anachronique (le baroque, stricto sensu, désigne un style du XVII. siècle) même si elle peut se révéler féconde au premier abord. Cf. Jean-Pierre Martel, "Trou de mémoire: œuvre baroque (essai sur le dédoublement et le décor)", Voix et images du pays VIII, Montréal, PUQ, 1974, p. 67-104.

48. Aquin s'est montré très conscient du fait que le livre est un objet matériel (un ensemble de feuilles pliées de façon à prendre tel format, cousues, brochees ou collées ensemble, reliées ou non, etc.) et que le texte ne se réduit pas à la somme de ses mots, qu'il comprend aussi la jaquette de couverture, la reliure, le type de papier, les illustrations (ou l'absence d'illustration), la typographie (type et corps de caractères, mise en page...), l'éditeur, la collection, le format... Ainsi, lire un ouvrage, quel qu'il soit, dans une collection de classiques, même pour la première fois, même si on ignorait auparavant jusqu'à son existence, c'est l'avoir en quelque façon déjà lu parce qu'il arrive porté par l'autorité de cette collection. De même, un in-folio massif (quelle metaphore dans le nom de la collection de poche publiée par les éditions Gallimard!) impose une tout autre attitude de lecture (mentale aussi bien que physique) qu'un petit format qu'on peut manipuler aisément, emporter avec soi en promenade, lire dans le métro. La couverture de Trou de mémoire, par exemple, qui reproduit une anamorphose de crâne, conduit tout droit au ccur du roman, vers le tableau d'Holbein, les Ambassadeurs, et la mort qui s'y tapit. De même, les arcs de triomphe renversés de la couverture de Points de fuite donnent le ton à ce 
recueil ironiquement narcissique, sorte d'entrée royale pompeuse et amusée de l'auteur Hubert Aquin.

49. Leonico Chigi publiera, en plus du Traité des maladies nouvelles, le journal de Jules-César Beausang dont il n'a pas pu avoir connaissance; lorsque JeanWilliam tente d'assassiner Robert, Christine reconnaît le revolver qui a servi au meurtre du pharmacien de San Diego auquel elle a assisté de très loin, de l'intérieur d'une voiture garée à une distance suffisante pour que Jean-William ne la remarque pas; elle quitte l'Hôpital de Cartierville à quatre heures du matin, immédiatement après avoir fait l'amour à sept heures du matin avec le docteur Franconi; Renata Belmissieri est tantôt une professionnelle de la contrebande, tantôt une novice... On pourrait multiplier les exemples, même sans tenir compte d'incroyables anachronismes.

50. Voir, à titre d'exemple, l'article de Jean Bélanger, “l'Antiphonaire", Études françaises, vol. VI, $n^{\circ} 2$, mai 1970, p. 217.

51. Hubert Aquin, «Plan partiel de l'Antiphonaire», op. cit., p. 104-105.

52. L'expression est d'Aquin lui-mème, dans un texte où, sans toutefois se référer expressément à l'Antiphonaire, il décrit en ces termes l'acte de lire: "Le lecteur tient le rôle d'officiant dans cette célébration; il officie en lisant le texte écrit, en se l'appropriant et en lui donnant - dans son for intérieur - un sens nouveau, une connotation et une dimension peut-être uniques n. Hubert Aquin, “la Disparition élocutoire du poète", op. cit., p. 8.

53. André Berthiaume, "le Roman (chronique)", Études françaises, vol. VI, $n^{\circ} 4$, novembre 1970, p. 499; repris dans Réal Ouellet (éd.), les Critiques de notre temps et le nouveau roman, op. cit., sous le titre "Un anti-antiphonaire" (p. 157-162). 